\title{
Mechanosensitivity of cell membrane may govern creep-strain recovery, osmotic expansion and lysis
}

\author{
Piotr H. Pawłowski ${ }^{\bowtie}$ \\ Institute of Biochemistry and Biophysics, Polish Academy of Sciences, Warszawa, Poland
}

Received: 26 May, 2009; revised: 30 August, 2009; accepted: 02 September, 2009

available on-line: 14 September, 2009

\begin{abstract}
A simple theoretical model considering cell membrane mechanosensitivity can accurately describe published experimental data on membrane area creeping and recovery, and on osmotic expansion and rupture. The model to data fit reveals real values of membrane tension and elasticity modulus, and the parameters describing membrane organization and kinetics of mechanosensitive membrane traffic, including small solute transport, water permeability, endocytosis, exocytosis, and caveolae formation. This estimation allows for separation and quantitative analysis of the participation of different processes constituting the response of plasmalemma to short time-scale membrane load. The predicted properties of the model were verified for membrane stretching at different osmotic pressures. Finally, a simple hypothesis concerning stressed cell membrane breakdown is postulated.
\end{abstract}

Keywords: cell membrane, surface tension, mechanosensitivity, endocytosis, exocytosis, caveolae

\section{INTRODUCTION}

During the last four decades many experiments of different type have been conducted to investigate the mechanical properties of the cell (Ananthakrishnan et al., 2006), and especially of the cell membrane, including: micropipette manipulation (Rand, 1964; Chien et al., 1978; Evans, 1983; Engström \& Meiselman, 1998; Hochmuth, 2000; Shao \& Xu, 2002; Ruef et al., 2004), cell poking (Daily et al., 1984; Duszyk et al., 1989; Goldmann, 2000), shear flow and fluid mechanical technique (Hochmuth et al., 1973; Pfafferott et al., 1985; Kon et al., 1987; Le et al., 1993; Firsov et al., 2006), osmotic expansion and lysis (Evans et al., 1976; Wolfe \& Steponkus, 1983; Wolfe et al., 1986; Dowgert et al., 1987), electric field elongation (Engelhardt \& Sackmann, 1988; Poznanski et al., 1992; Wanichapichart et al., 2002), magnetic bead microrheometry (Bausch et al., 1998; Wang et al., 2008), dynamic light scattering microscopy (Amin et al., 2007), optical tweezers (Hénon et al., 1999; Dao et al., 2003; Lian et al., 2004;), optical stretcher (Guck et al., 2000; 2001), microplate manipulation (Thoumine et al., 1999; Desprat et al., 2005), and microhand and atomic force microscopy (Yamashina \& Katsumata, 2000; Nishi et al., 2005). Some authors have proposed thermodynamics (Evans \& Skalak, 1980), pictorial spring-and-dashpot (Ehrenstein \& Iwasa, 1996; Moris et al., 2001), tensegrity (Wang et al., 2001), polymer network (Boal, 2002), bilayer-couple hypothesis (Lim et al., 2002), fluctuation (Fournier et al., 2004; Gov \& Safrany, 2005), statistical mechanics (Farago \& Pincus, 2004), Brownian dynamics (Noguchi \& Gompper, 2006), and advanced continuum mechanics ( $\mathrm{Tu}$, 2006) models to describe the rheological behavior of stretched biological material. This type of modeling assumes that the cell's response to stress, being in fact a static one, manifests both solid and fluid properties of the investigated entity. It directly reflects the specific structural arrangement of cellular material or simply resembles the response of a physical setup composed of some ideal elastic and viscous elements. Since the respective models usually neglect the lipid traffic and reorganization phenomena, the cellular membrane supported by the cytoskeleton is considered to be a closed and unchangeable ar-

${ }^{\square}$ Corresponding author: Piotr H. Pawłowski, Institute of Biochemistry and Biophysics PAS, A. Pawińskiego 5a, $02-106$ Warszawa, Poland; fax: (48) 3912 1623; e-mail: piotrp@ibb.waw.pl

Abbreviations: $B$, budding; $E n$, endocytosis; $E x$, exocytosis; $R$, cell radius; $t$, time, cell lifetime; $\tau$, tension; $S T r$, transport of small solute; WTr, transport of water. 
rangement. On the other hand, it is evident that the actual morpho-physiological knowledge related to the observed facts (Raucher \& Sheetz, 1999; Morris \& Homann, 2001; Apodaca, 2002; Ursell et al., 2008) calls for a more universal theoretical approach (Pawlowski, 2007), according to which the cell membrane should be characterized as a varying open mechanosensitive system, exchanging its material with the cytoplasm and the environment.

In this paper we propose a mechanokinetic approach dealing with endocytosis, exocytosis and caveolae invagination, which theoretically describes plasmalemma homeostasis during cell growth and division. This model considers transmembrane pressure, membrane tension and mechanosensitivity of membrane processes and meets the requirements of analysis of two groups of experiments in: $1-$ membrane area creep and recovery, 2 - membrane area osmotic expansion and cell lysis. Consistent literature data for micropipette elongation of membranes of rye protoplasts (Wolfe \& Steponkus, 1983) and for their osmotic deformation and rupture (Wolfe et al., 1986) were used as a pattern to be fitted by the model. To increase the generality of the results, these two experimental data sets were both fitted during the same fitting tour, preceded by simulation of a hypothetical cell cycle (for selection of consistent starting conditions). Thus, a single general setup of parameters was obtained covering three different areas of interest: a hypothetical cell cycle, forced elongation, and osmotic shock.

The resultant parameters describe the geometrical, compositional, mechanical, transport and self-organizational properties of rye protoplast cell membrane. Among others, they allow for a reliable estimation of the initial values of cell radius, membrane excluded area occupied by molecules, number of caveolae, surface tension, and elasticity modulus of the plasmalemma of the analyzed cells. The proposed model simulated experimentally forced evolution of the membrane area with satisfactory accuracy. Additionally, a quantitative analysis of the vesicular (exocytosis and endocytosis), bud-like (caveolae formation), and mechanical (stress-strain dependence) contributions to the cell membrane homeostasis indicates that during a short-time scale membrane load budding prevails over the vesicular effect only in the initial phase of stress action, whereas membrane extension due to mechanical stress dominates in osmotic expansion at all times.

The obtained model of a real cell membrane was positively verified from the point of view of its predictivity at different magnitudes and durations of osmotic stress.

The estimated increase in surface tension at the moment of membrane failure during osmotic shock allowed us to postulate a simple hypothesis regarding membrane breakdown in a stressed cell. This shows the utility of the model for theoretical considerations in similar conditions.

\section{THE MODEL}

\section{Basic equations}

A single cell was modeled as a mechanochemical set of plasmalemma, encapsulated cytoplasm and surrounding environment. It was assumed that membrane material can be delivered to the bilayer by exocytosis and removed by endocytosis. According to the model, a locally flat membrane can invaginate forming bud-like caveolae $(B)$. Single cell volume can change due to the transport of water $(W T r)$, small solute $(S T r)$, and endocytic $(E n)$ and exocytic $(E x)$ vesicles. The considered processes may be tension-sensitive $(\tau)$. For simplicity the encapsulated vesicle volumes as well as the excluded areas of the membrane, occupied by membrane material of endocytic and exocytic vesicles, were not distinguished. The vesicle and a cell volume exceeding certain border limit were treated as incompressible. Additionally, the shape of the cell was assumed to be spherical, and characterized by time $(t)$-dependent radius $R(t)$.

The basic equations of the model referring to the membrane structure and organization are presented below. They describe the kinetics of changes in the excluded cell membrane area, i.e., a geometrically occupied sectional area of all the molecules (lipids and proteins) of the membrane leaflet: and in the number of membrane buds:

$\frac{d A_{X}}{d t}=-A_{V X}\left(R_{E n}-R_{E x}\right)$ (Eqn. 1)

where: $t$ is time, $A_{X}$ - excluded area of the cell mem$\frac{d N_{B}}{d t}=\left(R_{B+}-R_{B-}\right)$

brane, $A_{V X}$ - membrane excluded area of endocytic and exocytic vesicles, $R_{E n^{\prime}} R_{E x^{\prime}} R_{B^{\prime}} R_{B-}-$ rates of endocytosis, exocytosis, budding and bud vanishing, $N_{B}$ - number of buds (caveolae).

In order to include mechanosensitivity, the rates: $R_{E n^{\prime}} R_{E x^{\prime}} R_{B y^{\prime}}$ and $R_{B-}$ were assumed to be tension-dependent. Exact formulas are given in Appendix A.

\section{Membrane area creep and recovery}

It was assumed that during an experiment on elongation and contraction of the cell, the membrane 
tension $\tau$ applied by a micropipette is kept constant in a stepwise manner:

$$
\tau=\tau_{i n i}+ \begin{cases}\Delta \tau_{1} & t>t_{1} \\ \Delta \tau_{2} & t>t_{2}\end{cases}
$$

where $\Delta \tau_{1}>\Delta \tau_{2}$.

To describe the mechanical properties of the cell membrane an equation describing the surface tension $\tau$ as:

$$
\tau=\tau_{0}-\frac{2 n A_{X} k_{B} T}{A-A_{X}}
$$

was added, where: $\tau_{0}$ is the surface tension at zero surface pressure, and the second term describes the pressure of membrane molecules behaving as a $2 \mathrm{D}$ gas. There, $n$ is the number of molecules per unit membrane (leaflet) excluded area, $k_{B}$ - Boltzmann constant, $T$ - temperature, and $A$ - total cell membrane area.

The total cell membrane area $A$ can be represented as:

$A=A_{P}+N_{B} \Delta A_{B}$

the sum of the projected membrane area, $A_{P}$, and the membrane area increase due to membrane budding, $N_{B} \Delta A_{B}$. There, $A_{P}$ is the area of an imagined smooth cell surface (without any invaginations) which corresponds to the average contour of the cell boundary visible with an optical microscope. Parameter $\Delta A_{B}$ describes the unprojected area of a single membrane bud.

Then, at a given $\tau$, according to Eqns. 4 and 5 , the evolution of projected membrane area $A_{P}$ is described by the equation:

$A_{P}=A_{X}+\frac{2 n A_{X} k_{B} T}{\tau_{0}-\tau}-N_{B} \Delta A_{B}$

where $A_{X}$ and $N_{B}$ are solutions of Eqns. 1 and 2.

\section{Osmotic expansion of membrane area and cell lysis}

It was assumed that during an experiment on expansion and rupture of the cell membrane, when the osmotic pressure of the environment abruptly changes from the initial isotonic value $p_{I}$ to the end value $p_{E^{\prime}}$ the transmembrane difference in the exerted osmotic pressure, $\Delta p_{O^{\prime}}$ varies as:

$\Delta p_{O}=p_{E}-p_{I} \frac{V_{I}}{V}$

where $V$ is the cell volume, starting with the initial value $V_{I}$.

At a given $p_{E}$, the evolution of the cell volume $V$ can be described by the equation: $\frac{d V}{d t}=L_{W T r} A_{X}\left(\Delta p_{H}-\Delta p_{O}\right)+k_{S T r} A_{X}+V_{V}\left(R_{E n}-R_{E x}\right)$

Here, $L_{W T r}$ is the hydraulic conductivity of the membrane, $\Delta p_{H}$ - external minus internal hydraulic pressure, $k_{S T r}$ - rate of small solute transport per unit of excluded membrane area, and $V_{V}$ - encapsulated volume of endocytic and exocytic vesicles. Tensiondependent rates $R_{E n}$ and $R_{E x}$ are given in Appendix A.

The general equation for the kinetics of cell volume variation (Eqn. 8), supplemented by equations describing the evolution of the excluded cell membrane area (Eqn. 1) and of the number of buds (Eqn. 2), and supported by equations calculating the surface tension (Eqn. 4), and the total cell membrane area (Eqn. 5), with the applied approximation

$A_{P}=(4 \pi)^{1 / 3}(3 V)^{2 / 3}$

give the rates of the considered processes and determine the considered variables.

\section{MATERIALS AND METHODS}

The analysis of membrane area creep and recovery relied on published data on micropipette aspiration of rye protoplasts (Wolfe \& Steponkus, 1983, Fig. 4). Similarly, in the analysis of osmotic expansion of membrane area and cell lysis data for osmotic deformation and rupture of rye protoplasts (Wolfe et al., 1986, Fig. 2a-f) were used.

Studies on the model of rye cell membrane involved the use of a two-step fitting procedure.

\section{First step - modeling a free cell}

In the first step of this procedure, for a proposed set of parameters, a hypothetical cell cycle was simulated according to a theoretical analysis of cell growth and division (briefly described in Appendix B). The objective was to obtain a repetitive course of variables: $A_{X}(t), N_{B}(t)$, and $V(t)$ meeting the assumed geometrical and mechanical requirements, and to select starting conditions for the next step of the procedure. The predefined requirements of this simulation were: cell radius, $R$, in the range $15<R<25 \mu \mathrm{m}$, and membrane tension, $\tau$, in the range $50<\tau<150 \mu \mathrm{N} / \mathrm{m}$ - the latter constraint required only in the tension-stable period of the cell cycle. Usually, a simulation embracing the history of three generations of cells was sufficient to obtain and confirm a stable and repetitive process of single cell growth and division. 


\section{Second step - modeling a loaded cell}

In the second step the proposed model was simultaneously fitted to two series of literature data. Two sets of equations were applied together: set \# 1 (Eqns. 1, 2 and 6) for membrane area creep and recovery, and set \# 2 (Eqns. 1, 2, 4, 5, 8 and 9) for osmotic expansion of membrane area and cell lysis. The starting values $A_{X}\left(t_{0}\right), N_{B}\left(t_{0}\right)$, and $V\left(t_{0}\right)$ were taken at an arbitrarily chosen moment $t_{0}$ of the tension-stable phase of the simulated cell cycle (first step of modeling) and the other parameters were left unchanged. In agreement with reported values on micropipette elongation and osmotic expansion, the difference $A_{P}-A_{P}\left(t_{0}\right)$ and the ratio $A_{P} /$ $A_{P}\left(t_{0}\right)$ were considered. The data for micropipette elongation were taken from a single-cell experiment. It was assumed: $\tau_{i n i}=125\left[\mu \mathrm{Nm}^{-1}\right], \Delta \tau_{1}=$ $1875\left[\mu \mathrm{Nm}^{-1}\right]$, and $\Delta \tau_{2}=-70\left[\mu \mathrm{Nm}^{-1}\right]$. Osmotic expansion data in an environment reduced from 0.53 to 0 Osm were taken from a multicell experiment, and before fitting they were averaged, including each cell before lysis.

\section{Calculations}

The above two-step procedure was repeated several times to obtain the parameters of the basic model $(B M)$ from the best fit between theory and experiment. Manually changed parameters at the beginning of each tour (before the first step) and the starting point $t_{0}$ (before the second step) were arbitrarily chosen according to literature findings, personal experience, and indications from previous simulations of the model. Numerical calculations were done in Mathematica 4.1 environment.

\section{Other simulations}

The analysis of bud, vesicle, and mechanical contributions to the variation in projected surface area increase, $\Delta A_{P}=A_{P}-A_{P}\left(t_{0}\right)$, during membrane load was performed using the finally accepted $B M$. The integrated "vesicle" and "bud" flows, $\Delta A_{P V}=\int_{t_{0}}^{t} A_{V X}\left(R_{E x}-R_{E n}\right) \mathrm{dt}^{\prime}$ and $\Delta A_{P B} \stackrel{=}{=} \int_{t_{0}}^{t} \Delta A_{B}\left(R_{B-}-R_{B+}\right) \mathrm{dt}^{\prime}$, and the net area difference, $\Delta A_{P M}=\Delta A_{P}-\Delta A_{P V^{-}}$ $\triangle A_{P B}$, were analyzed.

In testing the predictivity of the $B M$, the data for environment osmolarities ranging from 0 to 0.34 Osm and the stress exposure interval $t_{e}-t_{0}=30 \mathrm{~s}$, or $t_{e}-t_{0}=60 \mathrm{~s}$ were selected. Then, numerical predictions of the model were studied at recalculated values of osmotic pressure difference, $\Delta p_{o}\left(t_{0}\right)=p_{E}-p_{I}$, where $p_{I}=11.9$ [atm] $(0.53$ Osm), and other parameters taken from the accepted $B M$.

\section{RESULTS}

Fitting of the proposed model of the cell membrane to the data on micropipette elongation and osmotic deformation of rye protoplasts reveals, among other variables, variation in cell radius and membrane tension during the cell cycle (Fig. 1). During a long middle phase $(\Delta \tau \approx 500 \mathrm{~min})$ the cell radius increases, and then during mitosis the radii of separating hemispheres decrease (Fig. 1a). Moreover, a long middle phase of a relatively stable membrane tension can be clearly seen (Fig. 1b), indicating a favorable period for experimenting with cells.

The hypothetical starting conditions in the analyzed experiments with micropipette elongated and osmotically deformed cells were eventually selected at the 35th minute of the cell cycle, i.e., at the beginning of the middle phase (Fig. 2). This means that the studied cells contribute systems incorporating membrane material (Fig. 2a), budding (Fig. 2b), and growing (Fig. 2c) in a steady manner.

The accepted fit approximates the experimental pattern of variations in the projected surface area with satisfactory discrepancy that is below $10 \%$ (Fig. 3). It reveals asymmetry in the rate of membrane area creep and recovery (Fig. 3a). It also shows that osmotically stressed cells tend to,
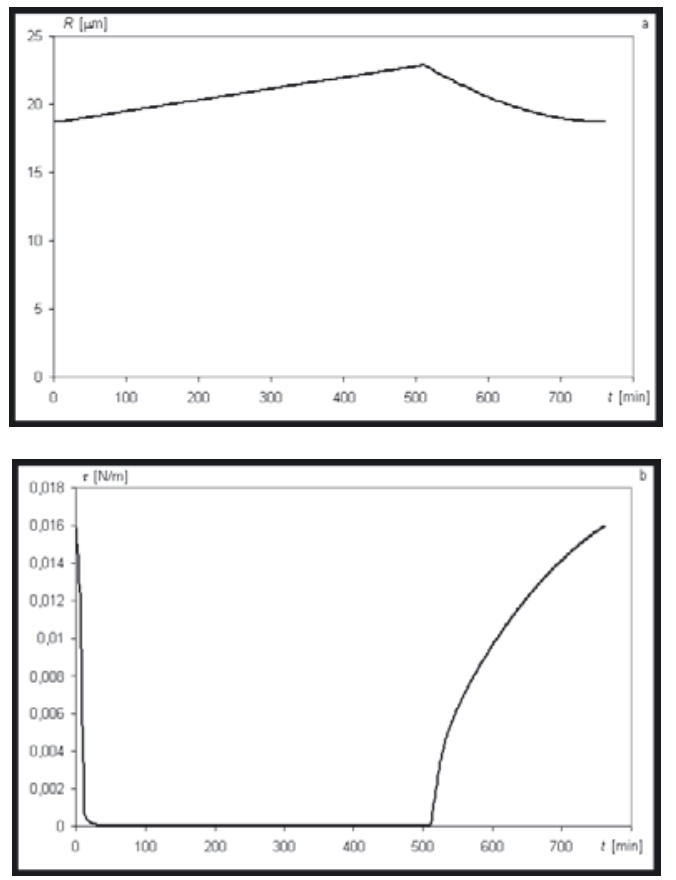

Figure 1. Fitting of the model (first step).

In the first step of each round of the fitting procedure (see Materials and Methods), simulation of the cell cycle passage with assumed geometrical and mechanical constrains was performed. Main results of the finally accepted simulation of the cycle are shown. a. Variations in cell radius, $R$. b. Variations in membrane tension, $\tau$. Symbol $t$ means cell lifetime. Optimized parameters are presented in Table 1. 

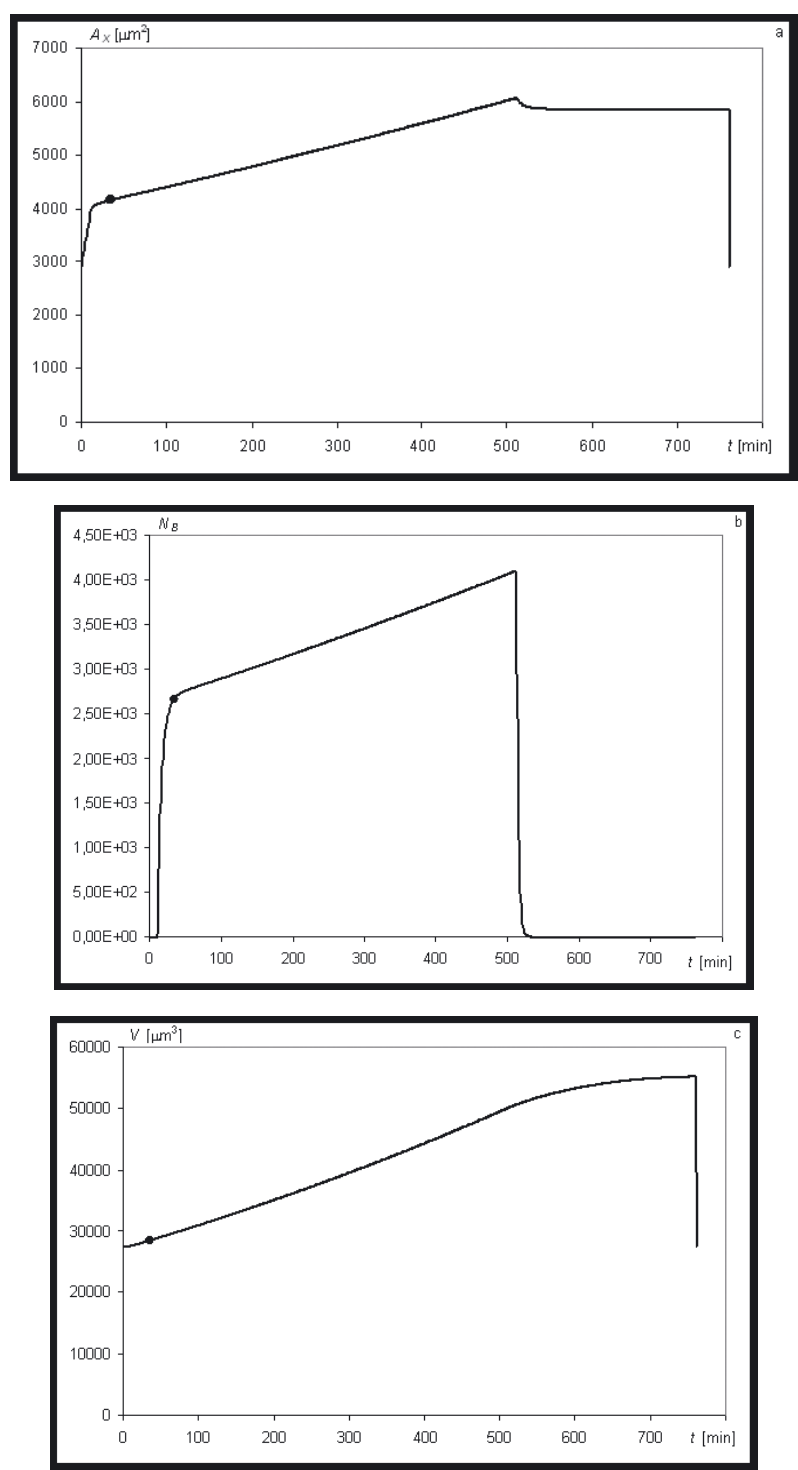

Figure 2. Fitting of the model (selected starting values). After each round of simulation of the cell cycle (see Fig. 1) and before the second step of the fitting procedure (see Materials and Methods), required starting values of membrane excluded area, $A_{x}\left(t_{0}\right)$, number of buds, $N_{B}\left(t_{0}\right)$, and cell volume, $V\left(t_{0}\right)$, were selected. Results of the finally accepted simulation of the cell cycle passage are shown, with the selected starting values for the second step of the procedure (filled points), taken at $t_{0}=35 \mathrm{~min}$. a. Variations in excluded membrane area, $A_{x^{\prime}}$ and selected $A_{x}\left(t_{0}\right)$. b. Variations in number of buds, $N_{B^{\prime}}$ and selected $N_{B}\left(t_{0}\right)$. c. Variations in cell volume, $V$, and selected $V\left(t_{0}\right)$. Other symbols and parameters as in Fig. 1 .

but do not reach, a stable mechanical balance under discussed conditions (Fig. 3b). The cell cycle and the experiments on area extension and cell lysis analyzed simultaneously can be described by a single set of parameters, shown in Table 1.

In general, the fitted model indicates the extent to which the vesicular processes, budding, and mechanical response (Fig. 4) contribute to the increase in surface area during membrane exposure
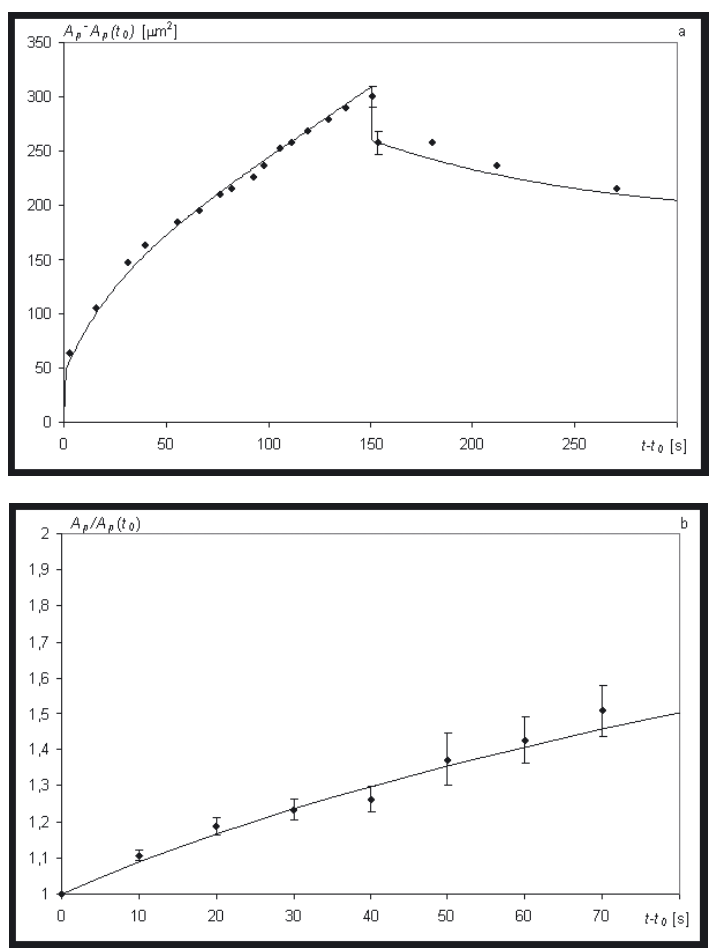

Figure 3. Fitting of the model (second step).

In the second step of each round of the fitting procedure (see Materials and Methods) the model was fitted to literature data. Results of the finally accepted fit are shown (continuous line). Here, $A_{p}$ is projected surface area, and $t-t_{0}$ is duration of experiment. Other symbols and parameters as in Figs. 1 and 2. a. Creep strain and recovery. Experimental points (Wolfe et al., 1983) were fitted using equation set \# 1. b. Osmotic expansion. Averaged experimental data (Wolfe et al., 1986) were fitted using equation set \# 2 .

to increased tension. It also shows that the relative magnitude of this participation depends on actual conditions. The dominance of the discussed components varies during micropipette elongation (Fig. 4a), and mechanical response dominates during osmotic expansion (Fig. 4b).

Predictions regarding the model of expansion of the membrane at different osmotic pressures and for a given exposure interval are in good agreement with the experimental data (Fig. 5). This agreement depends somewhat on the exposition time considered. In both analyzed cases (Figs. 5a and b) an approximately linear dependence of the degree of area expansion on the magnitude of the pressure can be observed.

\section{DISCUSSION}

The presented adjustment of the mechanokinetic model of micropipette elongation of the cell membrane and osmotic deformation of cells is an example of the universality of the discussed model and its parameters and of the concept of mechano- 

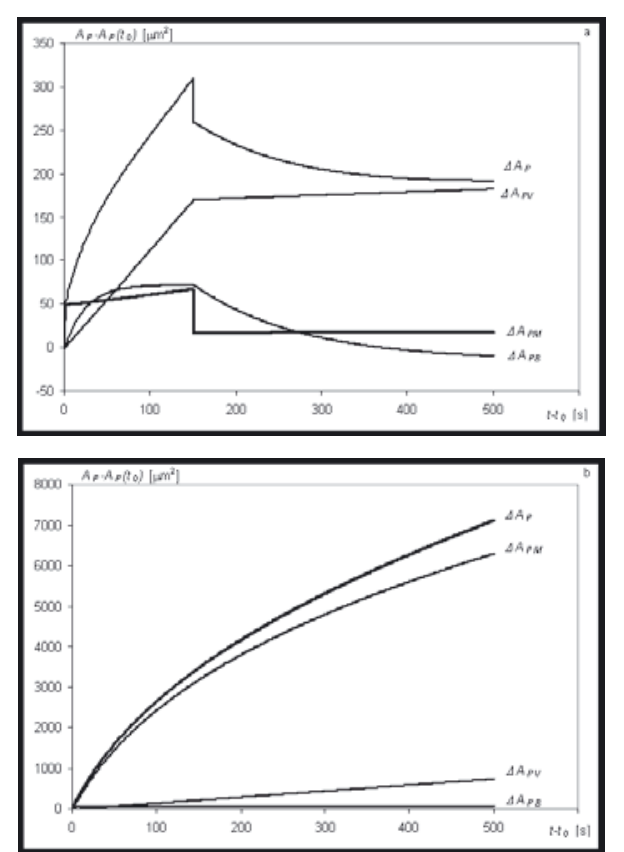

Figure 4. Processes constituting the response of plasmalemma to short time-scale membrane load according to the accepted model.

Variations in projected surface area increase, $\Delta A_{P}=A_{P}-$ $A_{P}\left(t_{0}\right)$, during membrane load are presented, with indicated contribution of vesicular $\left(\triangle A_{P V}\right)$, bud $\left(\triangle A_{P B}\right)$, and mechanical $\left(\triangle A_{P M}\right)$ component. a. Creep strain and recovery. b. Osmotic expansion. Other symbols and parameters as in Fig. 3.

sensitivity (Petrov \& Usherwood, 1994). The considered model includes the contribution of various phenomena to the observed response of the cell to stepstrain and osmotic shock. According to the author's knowledge it is the first one which appreciates the role of physiological processes such as membrane transport within the cell and membrane invaginations (caveolae) in the global mechanical state of the cell. It was shown that by applying the model and proposing a single set of parameters one can describe the course of the cell cycle (Figs. 1 and 2) and cell behavior in two different experimental situations (Fig. 3). The model describes the asymmetry in membrane area creep and recovery processes (Fig. 3a), and the exponential area increase under hypotonic pressure (Fig. 3b). Moreover, the model improves our knowledge on the participation of exocytosis, endocytosis, budding and mechanical stretching in the variation in membrane area exposed to stress (Fig. 4). As it was observed, mechanical stretching is the main process of membrane area modification at the beginning of cell elongation by means of a micropipette (Fig. 4a) as well as during osmotic expansion (Fig. 4b). Bud formation dominates over exocytosis only in the early stage of membrane load. The rate of vesicular support is strongly tension-dependent (Fig. 4a) and decreases with a decrease in
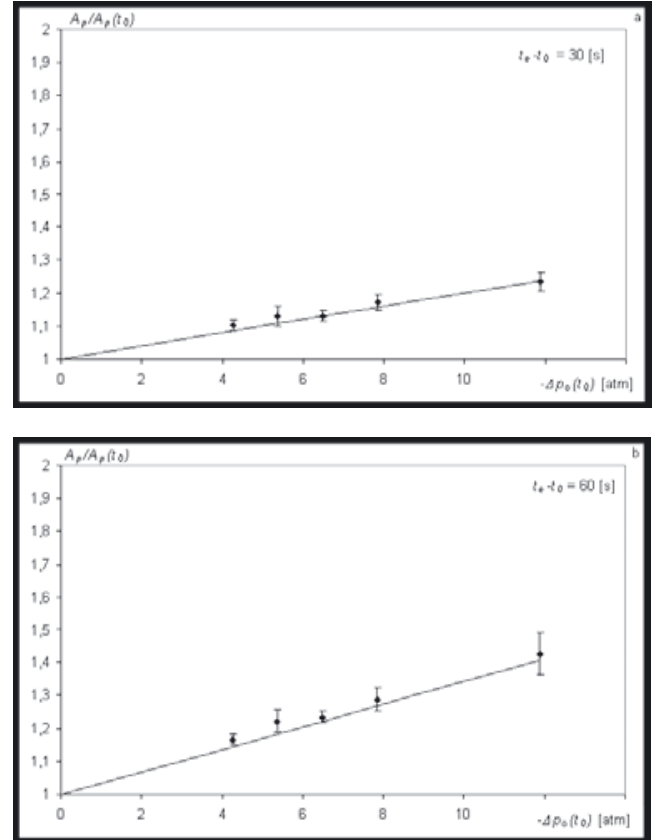

Figure 5. Predictions of the accepted model.

Shown is expansion of projected surface area, $A_{p}$, at different osmotic pressures, $\Delta p_{0^{\prime}}$ and for a given exposition interval, $t_{e}-t_{0}$. a. $t_{e}-t_{0}=30 \mathrm{~s}$. b. $t_{e}-t_{0}=60 \mathrm{~s}$. Continuous line was drawn according to equation set \#2 and parameters were taken from Table 1.

tension during membrane recovery. Under the same conditions bud vanishing prevails over bud formation. These findings argue for the role of mechanosensitivity in the cell response to stress. The predictive potential of the model is also worth underlining (Fig. 5), namely, its applicability to prediction of the expansion of the cell membrane at different osmotic pressures and exposition times.

The model can also be applied to the analysis of cell lysis observed during osmotic deformation experiments. Supposing that breakdown of the plasmalemma is related to mechanical energy (Pawlowski et al., 1993), in the case of osmotic stretching we may expect that membrane tension $\tau$ may

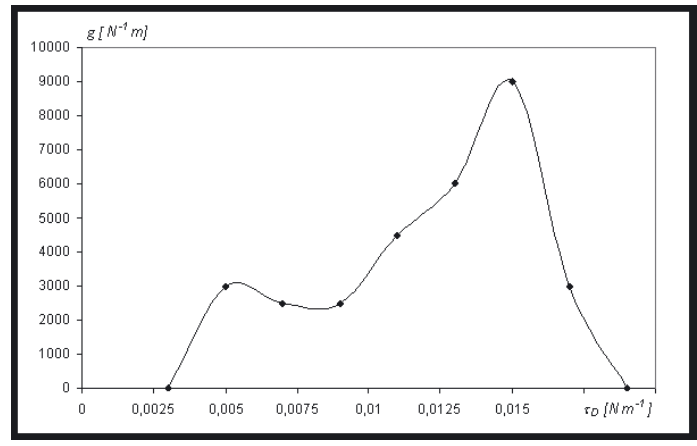

Figure 6. Membrane breakdown.

Density distribution, $g$, of destruction tension, $\tau_{D^{\prime}}$ in lysed cells, calculated according to the accepted model. Parameters were taken from Table 1. 
Table 1. Parameters of the accepted basic model

\begin{tabular}{|c|c|c|c|}
\hline \multicolumn{4}{|c|}{ Accepted basic model (BM) } \\
\hline Parameter & Value & Unit & Description \\
\hline$k_{B}$ & $1.381 \cdot 10^{-23}$ & $\mathrm{~J}^{\mathrm{o}} \mathrm{K}^{-1}$ & Boltzmann constant \\
\hline$T$ & 300 & ${ }^{\mathrm{o}} \mathrm{K}$ & temperature \\
\hline$\tau_{0}$ & $2 \cdot 10^{-2}$ & $\mathrm{~N} \mathrm{~m}^{-1}$ & surface tension at zero surface pressure \\
\hline$n$ & $2.5 \cdot 10^{-3}$ & $\AA^{-2}$ & no. of particles / excluded membrane area \\
\hline$\Delta p_{O}$ & $0^{*}$ & Atm & osmotic pressure difference \\
\hline$K_{V}$ & $10^{4}$ & Atm & cytoplasm volume compressibility \\
\hline$V_{E l}$ & $1.5 \cdot 10^{4}$ & $\mu \mathrm{m}^{3}$ & border value of cell volume \\
\hline$A_{v x}$ & $5 \cdot 10^{-2}$ & $\mu \mathrm{m}^{2}$ & excluded membrane area of vesicle \\
\hline$V_{V}$ & $2.5 \cdot 10^{-3}$ & $\mu \mathrm{m}^{3}$ & vesicle volume \\
\hline$\Delta A_{B}$ & $3 \cdot 10^{-2}$ & $\mu \mathrm{m}^{2}$ & unprojected area of bud \\
\hline$n_{B R}$ & 20 & $\mu \mathrm{m}^{-2}$ & no. of rafts / excluded membrane area \\
\hline$L_{W T r}$ & $8.5 \cdot 10^{-3}$ & $\mu \mathrm{m}$ atm $^{-1} \mathrm{~s}^{-1}$ & hydraulic conductivity of membrane \\
\hline$k_{S T r}$ & $1.5 \cdot 10^{-4}$ & $\mu \mathrm{m} \mathrm{s}^{-1}$ & rate of small solute transport \\
\hline$k_{E n}$ & $1.7 \cdot 10^{-3}$ & $\mu \mathrm{m}^{-2} \mathrm{~s}^{-1}$ & kinetic coefficient of endocytosis \\
\hline$k_{E x}$ & $1.75 \cdot 10^{-3}$ & $\mu \mathrm{m}^{-2} \mathrm{~s}^{-1}$ & kinetic coefficient of exocytosis \\
\hline$r_{E x}$ & 0 & & regulation factor \\
\hline$v_{B+}$ & $3 \cdot 10^{-4}$ & $\mathrm{~s}^{-1}$ & kinetic coefficient of budding \\
\hline$v_{B-}$ & $7.5 \cdot 10^{-3}$ & $\mathrm{~s}^{-1}$ & kinetic coefficient of bud vanishing \\
\hline$a_{E n}$ & 2.5 & $\mathrm{~nm}^{2}$ & geometric parameter of endocytosis \\
\hline$a_{E x}$ & 2.5 & $\mathrm{~nm}^{2}$ & geometric parameter of exocytosis \\
\hline$a_{B+}$ & 2.5 & $\mathrm{~nm}^{2}$ & geometric parameter of budding \\
\hline$a_{B-}$ & 3.5 & $\mathrm{~nm}^{2}$ & geometric parameter of bud vanishing \\
\hline$R_{E n L}$ & 30 & $\mathrm{~s}^{-1}$ & endocytosis rate limit \\
\hline$R_{E x L O}$ & 30 & $\mathrm{~s}^{-1}$ & regulated exocytosis rate limit \\
\hline$V(0)$ & $4 \pi R^{3}(0) / 3$ & & initial cell volume \\
\hline$R(0)$ & 18.744 & $\mu \mathrm{m}$ & initial cell radius \\
\hline$A_{X}(0)$ & 2919.546 & $\mu \mathrm{m}^{2}$ & initial excluded membrane area \\
\hline$N_{B}(0)$ & $2.129 \cdot 10^{-7}$ & & initial number of buds \\
\hline$Z(0)$ & 0 & $\mu \mathrm{m}$ & initial mitotic separation distance \\
\hline$V_{\min }$ & $5 \cdot 10^{4}$ & $\mu \mathrm{m}^{3}$ & minimum volume for mitosis \\
\hline$\tau_{\max }$ & $10^{-3}$ & $\mathrm{~N} \mathrm{~m}^{-1}$ & maximum tension for mitosis \\
\hline$Z_{\max }$ & $2 R$ & & maximum mitotic separation distance \\
\hline$u$ & $2.5 \cdot 10^{-3}$ & $\mu \mathrm{m} \mathrm{s}^{-1}$ & mitotic separation rate magnitude \\
\hline
\end{tabular}

*Value taken for cell cycle simulation

be an important parameter determining the process of cell lysis. For this reason, the distribution of the values of destruction tension, $\tau_{D^{\prime}}$ estimated for the membranes of lyzed cells, was analyzed (Fig. 6). The relevant values of tension were calculated for the moment of observed cell lysis, according to the model (set \# 2, Materials and Methods) and at osmotic pressures studied. The obtained results clearly indicate a consistent range of manifested destruction tensions of $0.003-0.019 \mathrm{Nm}^{-1}$, with a high distribution maximum near $\tau_{D}=0.015 \mathrm{Nm}^{-1}$. This confirms previous expectations and suggests similarity of the mechanical strength of different cells and biomembranes (Evans et al., 2003). In light of the above, the hypothesis that the value of the actual membrane tension may be one of important determinants of the lysis of rye protoplasts seems quite reasonable.

To confirm the applicability of the model to other cells and experimental techniques another fit was done to the results of an experiment on osmotic expansion of epithelial cells (Farinas \& Verkman, 1996). Only two parameters of the rye basic model, $L_{W T r}$ and $k_{S T r}$, had to be changed in order to obtain a satisfactory approximation (Fig. 7).

One may expect that the creep response of Fig. 3 could be reproduced quite easily with a viscoelastic dashpot-andsprings model containing much fewer parameters. Unfortunately, classical viscoelastic models do not describe the observed asymmetry in the rate of creep and recovery processes. Of course, such a model would not tell us anything about the cellular mechanisms controlling the viscoelastic parameters, a gap which the current model attempts to fill.

The cytoskeleton as such is absent from the presented modeling, while it is clear that although the membrane mechanics probably plays an important role in the overall cell mechanics, many features of the cell's mechanical response may be dominated by the cytoskeleton (Sikorski et al., 2000). This problem requires further investigation. Furthermore, the fitting procedure and

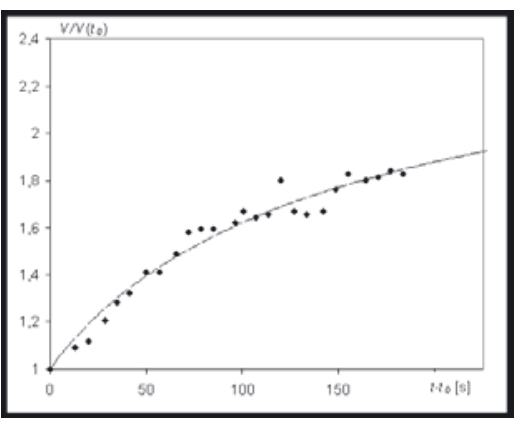

Figure 7. Osmotic expansion of MDCK cells.

Experimental data (Farinas et al., 1996) were fitted using equation set \# 2 and the parameters taken from Table 1 , with modified values of $L_{W T r}\left(2.5\right.$-fold increase) and $k_{S T r}$ (50-fold increase). 


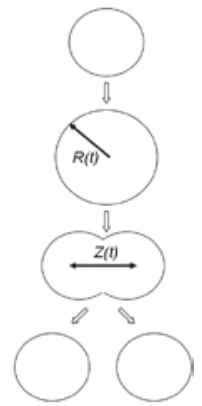

Figure 8. Theoretical model of cell growth and division.

Geometry of the system. $R(t)$ is radius of the cell or the mitotic cut sphere, $Z(t)$ is mitotic separation distance.

the criteria adopted to choose the best set of parameters need some improvement.

The examples presented in this paper represent positive tests of the validity of the mechanokinetic approach to the cell membrane. In future, it is expected that the discussed approach, due to its generality, can also be adapted to describe many other experimental and physiological situations, especially involving protoplasts or cells without a complex cytoskeleton and extracellular matrix.

\section{APPENDIX A}

Calculation of $R_{E n^{\prime}} R_{E x^{\prime}} R_{B^{+}}$and $R_{B-}$

The rates of endocytosis, $R_{E n^{\prime}}$ exocytosis, $R_{E x^{\prime}}$ bud formation, $R_{B^{+}}$and bud vanishing, $R_{B-}$, were calculated as:

$R_{E n}=\operatorname{Min}\left[k_{E n} \operatorname{Exp}\left(\frac{-a_{E n} \tau}{K_{B} T}\right) A_{X}, R_{E n L}\right]$

(Eqn. 10)

$R_{E x}=\operatorname{Min}\left[k_{E x} \operatorname{Exp}\left(\frac{a_{E x} \tau}{K_{B} T}\right) A_{X}, R_{E x L}\right]$

$R_{B+}=v_{B+} \operatorname{Exp}\left(\frac{-a_{B+} \tau}{K_{B} T}\right)\left(n_{B R} A_{X}-N_{B}\right)$

$R_{B-}=v_{B-} \operatorname{Exp}\left(\frac{a_{B-} \tau}{K_{B} T}\right) N_{B}$

where: Min is an operator returning the smaller of the two values, $k_{E n^{\prime}} k_{E x^{\prime}} v_{B_{+}}$and $v_{B_{-}}$are the kinetic coefficients of endocytosis, exocytosis, budding and bud vanishing, respectively, describing possible membrane activity in a given process at zero membrane tension, $a_{E n^{\prime}} a_{E x^{\prime}} a_{B^{+}}$and $a_{B-}$ are the geometric parameters describing some energetically important changes in membrane area during a given process, $R_{E n L}$ and $R_{E x L}$ are extramembrane limits in the rate of the considered processes constrained by the efficiency of vesicle recycling, metabolism and regulation, whereas $n_{B R}$ is the number of budding regions (rafts) per unit of excluded membrane area. Similar approach was taken by Sens and Turner (2006).

\section{APPENDIX B}

\section{Cell growth and division}

A growing cell was assumed to be spherical in shape (Fig. 8), with a time $(t)$-dependent radius $R(t)$. During mitosis the cell rearranges its geometry forming a two-kernel peanut shape, made of a symmetrical pair of cut and joined spheres, with the centers separated by a distance $Z(t)$ shorter than the sum of radii of sister halves.

Cell cycle was simulated according to the set of equations:

$\frac{d A_{X}}{d t}=-A_{V X}\left(R_{E n}-R_{E x}\right)$

$\frac{d N_{B}}{d t}=\left(R_{B+}-R_{B-}\right)$

(Eqn. 15)

$\frac{d V}{d t}=L_{W T r} A_{X}\left(\Delta p_{H}-\Delta p_{O}\right)+k_{S T r} A_{X}+V_{V}\left(R_{E n}-R_{E x}\right)$

$\frac{d Z}{d t}=u_{Z}$

where $Z$ is the mitotic separation distance between the centers of the daughter cells during division, and $u_{Z}$ is the mitotic separation rate. The meaning of other symbols is the same as in the main text and in Appendix A.

The defined geometry of the system relates the unknown total membrane area $A$ to the cell volume $V$, the mitotic separation distance $Z$, and the number of caveolae $N_{B}$. One can write:

$A=4 \pi R^{2}\left(1+\frac{Z}{2 R}\right)+N_{B} \Delta A_{B}$

(Eqn. 18)

where $R$ is the cell radius and also the mitotic cut sphere radius, which can be calculated as:

$R=\sqrt[3]{\frac{1}{2}\left(\frac{Z^{3}}{32}+\frac{3}{4 \pi} V\right)+\frac{1}{2} \sqrt{\left(\frac{Z^{3}}{32}+\frac{3}{4 \pi} V\right)^{2}-\frac{Z^{6}}{32^{2}}}}$

$+\sqrt[3]{\frac{1}{2}\left(\frac{Z^{3}}{32}+\frac{3}{4 \pi} V\right)-\frac{1}{2} \sqrt{\left(\frac{Z^{3}}{32}+\frac{3}{4 \pi} V\right)^{2}-\frac{Z^{6}}{32^{2}}}}-\frac{Z}{4}$

(Eqn. 19)

For a growing spherical cell Eqn. 19 simply gives the expected value $R=(3 V / 4 \pi)^{1 / 3}$.

Surface tension $\tau$ was calculated as:

$\tau=\tau_{0}-\frac{2 n A_{X} k_{B} T}{A-A_{X}}$

(Eqn. 20)

Mechanical balance approximation led to the description of the hydraulic pressure difference across the membrane $\Delta p_{H}$ by Laplace law combined with ideal elasticity law:

$\Delta p_{H}=-\frac{2 \tau}{R}-K_{V} \frac{\left(V-V_{E l}\right)}{V_{E l}} \Theta\left(V_{E l}-V\right)$

(Eqn. 21) 
Here, $K_{v}$ is the modulus of cytoplasm volume compressibility, $V_{E l}$ - border value of the cell volume for the activation of elastic cytoplasmic response, $\Theta-$ a unit step Heaviside function. In the above, the second component describes an additionally activated elastic response of the cytoplasm, when the cell volume decreases below a certain value $V_{E l}$.

We assumed that a cell initiates mitotic separation when its volume reaches a certain minimum value $V_{\text {min }}$ and the membrane tension is not higher than a certain critical value $\tau_{\max }$ so:

$u_{Z}=u \Theta\left(V-V_{\min }\right) \Theta\left(\tau_{\max }-\tau\right)$

where $u$ is the separation rate magnitude.

To model the regulation of exocytosis rate we assumed that

$R_{E x L}=\left\{\begin{array}{ccc}R_{E x L 0} & \text { for } & u_{z}=0 \\ r_{E x} R_{E x L 0} & \text { for } & u_{z}>0\end{array}\right.$

where $R_{E x L 0}$ is a constant value, $r_{E x}-$ a regulation factor.

The cell divides symmetrically when $Z$ exceeds a certain maximal value $Z_{\max }$. Then it is algorithmically imputed:

$\left\{\begin{array}{c}V=V / 2 \\ A_{X}=A_{X} / 2 \\ N_{B}=N_{B} / 2 \\ Z=0\end{array}\right.$

(Eqn. 24)

\section{REFERENCES}

Amin MS, Park YK, Ramachandra NL, Dasari R, Badizadegan K, Feld MS, Popescu G (2007) Microrheology of red blood cell membranes using dynamic scattering microscopy. Opt Express 15: 17001-17009.

Ananthakrishnan R, Guck J, Käs J (2006) Cell mechanics: recent advances with a theoretical perspective. Recent Res Devel Biophys 5: 39-69.

Apodaca G (2002) Modulation of membrane traffic by mechanical stimuli. Am J Physiol Renal Physiol 282: F179F190.

Bausch AR, Ziemann F, Boulbitch AA, Jacobson K, Sackmann E (1998) Local measurements of viscoelastic parameters of adherent cell surfaces by magnetic bead microrheometry. Biophys J 75: 2038-2049.

Boal D (2002) Mechanics of the Cell. University Press, Cambridge.

Chien S, Sung KLP, Skalak R, Usami S (1978) Theoretical and experimental studies on viscoelastic properties of erythrocyte membrane. Biophys J 24: 463-478.

Daily B, Elson EL, Zahalak GI (1984) Cell poking. Determination of the elastic area compressibility modulus of the erythrocyte membrane. Biophys J 45: 671-682.

Dao M, Lim CT, Suresha S (2003) Mechanics of the human red blood cell deformed by optical tweezers. J Mech Phys Solids 51: 2259-2280.

Desprat N, Richert A, Simeon J, Asnacios A (2005) Creep function of a single living cell. Biophys J 88: 2224-2233.
Dowgert MF, Wolfe J, Steponkus PL (1987) The mechanics of injury to isolated protoplasts following osmotic contraction and expansion. Plant Physiol 83: 1001-1007.

Duszyk M, Schwab B, Zahalak GI, Qian H, Elson EL (1989) Cell poking: quantitative analysis of indentation of thick viscoelastic layers. Biophys J 55: 683-690.

Ehrenstein D, Iwasa KH (1996) Viscoelastic relaxation in the membrane of the auditory outer hair cell. Biophys J 71: 1087-1094.

Engelhardt H, Sackmann E (1988) On the measurement of shear elastic moduli and viscosities of erythrocyte plasma membranes by transient deformation in high frequency electric fields. Biophys J 54: 495-508.

Engström KG, Meiselman HJ (1998) Effects of pressure on red blood cell geometry during micropipette aspiration. Cytometry 23: 22-27.

Evans EA, Waugh R, Melnik L (1976) Elastic area compressibility modulus of red cell membrane. Biophys $J$ 16: 585-595.

Evans EA, Skalak R (1980) Mechanics and Thermodynamics of Biomembranes. CRC Press, Boca Raton, FL.

Evans EA (1983) Bending elastic modulus of red blood cell membrane derived from buckling instability in micropipet aspiration tests. Biophys J 43: 27-30.

Evans E, HeinrichV, Ludwig F, Rawicz W (2003) Dynamic tension spectroscopy and strength of biomembranes. Biophys J 85: 2342-2350.

Farago O, Pincus P (2004) Statistical mechanics of bilayer membrane with a fixed projected area. J Chem Phys 120: 2934-2950.

Farinas J, Verkman AS (1996) Cell volume and plasma membrane osmotic water permeability in epithelial cell layers measured by interferometry. Biophys J 71: 35113522.

Firsov NN, Priezzhev AV, Klimova NV, Tyurina AY (2006) Fundamental laws of the deformational behavior of erythrocytes in shear flow. J Eng Phys Thermophys 79: 118-124.

Fournier JB, Lacoste D, Raphael E (2004) Fluctuation spectrum of fluid membranes coupled to an elastic meshwork: jump of the effective surface tension at the mesh size. Phys Rev Lett 92: 018102.1-018102.4.

Goldmann WH ( 2000) Mechanical manipulation of animal cells: cell indentation. Biotech Lett 22: 431-435.

Gov NS, Safrany SA (2005) Red blood cell membrane fluctuations and shape controlled by ATP-induced cytoskeletal defects. Biophys J 88: 1859-1874.

Guck J, Ananthakrishnan R, Moon TJ, Cunningham CC, Kas J (2000) Optical deformability of soft biological dielectrics. Phys Rev Lett 84: 5451-5454.

Guck J, Ananthakrishnan R, Mahmood H, Moon TJ, Cunningham CC, Kas J (2001) The optical stretcher: a novel laser tool to micromanipulate cells. Biophys J 81: 767784.

Hénon S, Lenormand G, Richert A, Gallet F (1999) A new determination of the shear modulus of the human erythrocyte membrane using optical tweezers. Biophys J 76: 1145-1151.

Hochmuth RM, Mohandas N, Blackshear PL Jr (1973) Measurement of the elastic modulus for red cell membrane using a fluid mechanical technique. Biophys J 13: 747-762.

Hochmuth R (2000) Micropipette aspiration of living cells. J Biomech 33: 15-22.

Kon K, Maeda N, Shiga T (1987) Erythrocyte deformation in shear flow: influences of internal viscosity, membrane stiffness, and hematocrit. Blood 69: 727-734.

Le KH, Quan S, Levenson J, Pino MD, Simon A, Devynck MA (1993) In vivo shear flow and erythrocyte mem- 
brane fluidity in hypertensive patients. $\mathrm{Br} J$ Clin Pharmacol 36: 437-443.

Lian GH, Xiang LC, Fa DJ, Qiang JY, Hai HX, Lin LZ, Ying CB, Zhong ZD (2004) Mechanical properties of breast cancer cell membrane studied with optical tweezers. Chinese Phys Let 21: 2543-2546.

Lim GHW, Wortis M, Mukhopadhyay R (2002) Stomatocyte-discocyte-echinocyte sequence of the human red blood cell: Evidence for the bilayer-couple hypothesis from membrane mechanics. Proc Natl Acad Sci USA 99: 16766-16769.

Morris CE, Homann U (2001) Cell surface area regulation and membrane tension. J Membr Biol 179: 79-102.

Nishi D, Arai T, Inoue K, Takubo T (2005) Measurement of the mechanical properties of living cell using micro hand and developed AFM system. In: Proceedings of IEEE/RSJ International Conference on Intelligent Robots and Systems, 2-6 Aug. 2005, pp. 990-995.

Noguchi H, Gompper G (2006) Meshless membrane model based on the moving least-squares method. Phys Rev 73: 021903. 1-021903. 12.

Pawlowski PH (2007) Mechanokinetic model of cell membrane; theoretical analysis of plasmalemma homeostasis, growth and division. J Theor Biol 249: 67-76.

Pawlowski P, Szutowicz I, Marszalek P, Fikus M (1993) Bioelectrorheological model of the cell. 5. Electrodestruction of cellular membrane in alternating electric field. Biophys J 65: 541-549.

Petrov AG, Usherwood PNR (1994) Mechanosensitivity of cell membranes. Eur Biophys J 23: 1-19.

Pfafferott C, Nash GB, Meiselman HJ (1985) Red blood cell deformation in shear flow. Effects of internal and external phase viscosity and of in vivo aging. Biophys J 47: 695-704.

Poznanski J, Pawlowski P, Fikus M (1992) Bioelectrorheological model of the cell. 3. Viscoelastic shear deformation of the membrane. Biophys J 61: 612-620.

Rand RP (1964) Mechanical properties of the red cell membrane. II. Viscoelastic breakdown of the membrane. Biophys J 4: 303-316.

Raucher D, Sheetz MP (1999) Characteristics of a membrane reservoir buffering membrane tension. Biophys J 77: 1992-2002.

Ruef P, Pöschl JMB, Linderkamp O (2004) Formation and relaxation of erythrocyte membrane tethers in micropipettes. Clin Hemorheol Microcirc 30: 39-46.
Sens P, Turner MS (2006) Budded membrane microdomains as tension regulators. Phys Rev E 73: 031918.

Shao JY, Xu J (2002) A modified micropipette aspiration technique and its application to tether formation from human neutrophils. J Biomech Eng 124: 388-397.

Sikorski AF, Hanus-Lorenz B, Jezierski A, Dlużewski AR (2000) Interaction of membrane skeletal proteins with membrane lipid domain. Acta Biochim Polon 47: 565578.

Thoumine O, Ott A, Cardoso O, Meister JJ (1999) Microplates: a new tool for manipulation and mechanical perturbation of individual cells. J Biochem Biophys Methods 39: 47-62.

Tu ZC (2006) Elastic theory of membranes. AAPPS Bull 16: 30-33.

Ursell T, Phillips R, Kondev J, Reeves D, Wiggins PA (2008) The role of lipid bilayer mechanics in mechanosensation. In: Mechanosensitive Ion Channels, pp 37-70 Springer, Netherlands.

Wang N, Naruse K, Stamenović D, Fredberg JJ, Mijailovich SM, Tolić-Nørrelykke IM, Polte T, Mannix R, Ingber DE (2001) Mechanical behavior in living cells consistent with the tensegrity model. Proc Natl Acad Sci USA 98: 7765-7770.

Wang CC, Jian HJ, Wu CW, Lee CH (2008) Cell membrane deformations under magnetic force modulation characterized by optical tracking and non-interferometric widefield profilometry. Microsc Res Techn 71: 594-598.

Wanichapichart P, Maswiwat K, Kanchanapoom K (2002) Elastic constant of Dendrobium protoplasts in AC electric fields. Membr Sci Tech 24: 799-806.

Wolfe J, Steponkus PL (1983) Mechanical properties of the plasma membrane of isolated plant protoplasts. Mechanism of hyperosmotic and extracellular freezing injury. Plant Physiol 71: 276-285.

Wolfe J, Dowgert MF, Steponkus PL (1986) Mechanical study of the deformation and rupture of the plasma membranes of protoplasts during osmotic expansions. J Membr Biol 93: 63-74.

Yamashina S, Katsumata O (2000) Structural analysis of red blood cell membrane with an atomic force microscope. J Electron Microsc 49: 445-451. 